\title{
Stand und Perspektiven der Versicherungswirtschaft in Polen
}

\author{
von Andrej Gawronski*
}

\section{1. "Durchbruch" im polnischen Versicherungswesen}

Von einem "Durchbruch" in der polnischen Versicherungswirtschaft schrieb man in der Nachkriegsfachliteratur - auch in der westlichen - mindestens zweimal, indem man mit diesem Begriff die Reformen am Ausgang der fünfziger Jahre ${ }^{\prime}$ und um die Mitte der achtziger bezeichnete. ${ }^{2}$ Gelegentlich komplimentierte man polnische Regelungen, einerseits für ihre Originalität und Fortschrittlichkeit im Vergleich zu den anderen damals geltenden Systemen in Mittel- und Osteuropa, andererseits - für deren Verbindung mit ihren Wurzeln und im allgemeinen - mit der Versicherungstradition.

Ich glaube jedoch, dass die aufmerksamen Beobachter der Wirtschaftsbühne dieser Region Europas die Meinung teilen werden, dass die zur Zeit in Polen im Bereich der Versicherungen unternommenen Versuche - die nur ein Fragment eines komplexen Programms der wirtschaftlichen Umwandlungen sind -, eine im vollen Sinne des Wortes tatsächliche Wende in diesem Sektor der wirtschaftlichen Aktivität verkündigen.

Über 40 Jahre hindurch wurden die Versicherungen (genauer das Versicherungsangebot) an eine im ökonomischen Sinn unwirkliche Umgebung (die zentralgeplante Wirtschaft) durch einen Monopolisten - der sich eines in der Marktwirtschaft bewährten Versicherungsmechanismus bediente - adressiert. Diese "Unechtheit der Umgebung" beruhte darauf, dass die in die Wirtschaftprozesse engagierten Subjekte in Anlehnung an die mit dem Wertgesetz inkohärenten Parameter (Preise, Löhne, Prozentsatz usw.) sowie an die anders als die Marktbedingungen gearteten Motivationen ("das allgemeine Wohl", "Verknüpfung des individuellen mit dem gesellschaftlichen Interessc" u. ä.) gewirkt haben.

Ein ähnlich verzerrtes Parameter war unter diesen Bcdingungen das Wirtschaftsrisiko.

Im Jahre 1984 hat man den Versuch unternommen, die Versicherungswirtschaft zu demonopolisieren, indcm man durch Gesctz erlaubtc, anderc - neben der bislang einzigen Versicherungsanstalt Panśtwowy Zaklad Ubezpieczeń (PZU) - Versicherungsunternehmen

\footnotetext{
"Poznan.
}

${ }^{1}$ Es ging hier um die Einführung der damals in der sozialistischen Wirtschaft kontroversen Versicherungen des Vermögens der staatlichen Betriebe und die rasche Entwicklung der Versicherungen des privaten Sektors.

${ }^{2}$ Gesetz über Vermögens- und Personenversicherungen von 1984. 
im Rahmen des sog. vergesellschafteten Sektors (d. i. des staatlichen und genossenschaftlichen Eigentums) zu gründen. Infolge dessen ist eine genossenschaftliche Versicherungsanstalt ("Westa" in Lódź) entstanden, die jedoch eine nur geringe wirtschaftliche Bedeutung hat.

Auf dem Gebiet der Tätigkeit der Hauptabnehmer von Versicherungsdienstleistungen erfolgten allerdings de facto keine Veränderungen: die von Zeit zu Zeit verkündeten "Reformen" im Funktionieren der Staatsbetriebe hatten - angesichts des Mangels an wesentlichen politischen Veränderungen - nur eine kosmetische, äusserliche Bedeutung.

Die seit Juni letzten Jahres in Polen stattfindenden Umgestaltungen sowie die als ihre Folgen auftretenden Veränderungen der Wirtschaftsordnung schaffen Grundlagen für die Einrichtung in diesem Lande eines Versicherungssystems, das sich selbst auf die Grundsätze des freien Wettbewerbs stützt und seine Dienstleistungen an die Subjekte adressiert, die unter wirklichen Marktbedingungen funktionieren. Es wurde in dieser Richtung ein begrüssenswerter Schritt nach vorne getan.

Die Grundlagen für ein neues Versicherungssystem soll eines der jüngsten von dem Sejim beschlossenen Gesetze - das Gesetz vom 28. 7. 1990 über die Versicherungstätigkeit, das auch an diesem Tag in Kraft getreten ist - schaffen.

\section{Die neue Regelung im Bereich der Versicherungen}

Schon am Anfang ist zu bemerken, dass das oben zitierte Gesetz die der EG angenäherten Teillösungen praktisch auf allen Regelungsebenen ${ }^{3}$ vorsieht. Diese Nachbildung resultiert sowohl aus dem Willen, die Erfahrungen auszunutzen und die bewährten Muster zu adaptieren, als auch aus den öffentlich deklarierten Bemühungen, den Status eines mit dem Gemeinsamen Markt assoziierten Mitgliedes zu erhalten. ${ }^{4}$

Das von der neuen Regelung umfasste Problemfeld steht natürlich in keinem Verhältnis zu den bisherigen Gesetzen, die die Tätigkeitsbedingungen eines einzigen staatlichen Versicherers, der ein direkt mit dem Finanzsystem des Staates (Staatshaushalt, Monobank) verbundenes Glied darstellt, bestimmt hatten . somit:

$\mathrm{Zu}$ den wichtigsten neuen - wenigstens in der Nachkriegszeit - Lösungen gehören

1. die Öffnung des Zugangs zu dem polnischen Versicherungmarkt für neue polnische Versicherer - und vor allem - für ausländische Versicherer;

2. die Einführung der einheitlichen Grundsätze der Konzessionierung der Versicherungstätigkeit,

(u. a. die Bestimmung der Kapital-, Eignungs- und Organisationserfordernisse für diejenigen Subjekte, die sich um die Erteilung einer Genehmigung zur Führung einer Versicherungstätigkeit bewerben, sowie die Grundsätze der Finanzwirtschaft);

3. die Einführung der Institution der Versicherungsaufsicht;

${ }^{3}$ Siche: Begründung zum Gesetz über Versicherungstätigkeit vom 28. 8. 1990 - Vervielfältigungsdruck des Parlaments (1990, August - ohne Nummer).

¿ Man soll dabei betonen, dass der Gesetzgeber sich für ein System der "finanziellen Regelung" der Versicherungswirtschaft entschieden hat, das von der EG-Kommission empfohlen wird und sich dem britischen Versicherungsaufsichtsystem nähert. 
4. die Beschränkung der Rechtsformen der Versicherungsunternehmen auf Aktiengesellschaften und Versicherungsgesellschaften auf Gegenseitigkeit;

5. die Beschränkung des Umfanges der Wirtschaftstätigkeit der Versicherer auf die strikte Versicherungstätigkeit;

6. die Einführung des Verbotes einer gleichzeitigen Aufnahme der Tätigkeit im Bereich der Lebensversicherungen und der sonstigen Versicherungsarten;

7. die Einführung der Grundsätze des Konkursverfahrens und der Liquidierung der Versicherungsanstalten;

8. die Liquidierung der obligatorischen (sog. gesetzlichen) Versicherungen mit der Ausnahme: der Kfz-Haftpflicht-, Gebäude-Feuerversicherungen in der Landwirtschaft und Landwirt-Haftpflichtversicherungen);

9. die Errichtung eines Sonderfonds für den Schutz der Interessen der Opfer bei Unfällen, die durch die obligatorischen Versicherungen nicht umfasst wurden, und für den Fall der Erklärung der Zahlungsunfähigkeit durch den Versicherer.

Die dekretierte Rechtslösung schafft den Rahmen für die Allgemeinheit der Subjekte, die an der Versicherungstätigkeit interessiert sind, entscheidet aber natürlich nicht darüber, ob diese Chance wahrgenommen wird. Deren Nutzung hängt einerseits davon $a b$, ob die Wirtschaftssubjekte (polnische) über ein ausreichendes Finanz-, Organisations- und Kaderpotential verfügen, and andererseits davon, wie gross das wirkliche Nachfragepotential ist, das von der Allgemeinheit der am Versicherungsschutz interessierten Subjekte vertreten wird.

An dieser Stelle möchte ich auf einen Kommentar zu den Bestimmungen des Gesetzes verzichten und mich auf die Frage der Einschätzung der heutigen Lage und Perspektiven des polnischen Versicherungsmarktes im Bereich des Angebotes an Dienstleistungen und der Nachfrage nach ihm konzentrieren.

\section{Die aktuelle Angebotsstruktur des Versicherungsmarktes in Polen}

\section{I. Die Struktur der Versicherungsunternehmen}

Auf dem polnischen Versicherungsmarkt hat sich zur Zeit folgende Struktur der Versicherungsunternehmen gestaltet:

1. Es dominiert entschieden der frühere Monopolist, die PZU ("nicht aus eigenem Willen", wie oft betont wird), die ungefähr $98 \%$ der allgemeingesammelten Versicherungsbeiträge im Lande umfasst;

2. Sie wird von einer anderen, ehemals auch monopolistischen Versicherungsanstalt "Warta" A.G. begleitet (eine typische Struktur der Versicherungswirtschaft für die Zeit der zentralgeplanten Wirtschaft). In dieser Hinsicht hat sie weiterhin eine marktbeherrschende Stellung (mit der Ausnahme von touristischen Versicherungen). Die "Warta" hat dagegen noch nicht in einem grösseren Ausmass die Versicherung von inländischen Risiken übernommen, obwohl sie rechtlich mit einer solchen Möglichkeit ausgestattet ist.

3. Neben der PZU existiert auf dem Markt der inländischen Risiken eine Gruppe von kleineren Versicherungsfirmen. Ihre Entstehung ist eine Folge der Übergangsperiode zwischen der demonopolisierenden Regelung aus dem Jahre 1984, einer Reihe von 
Wirtschaftsgesetzen von 1989-90, die die Möglichkeit wiederhergestellt haben, dass die Wirtschaftstätigkeit durch Privatpersonen in Form von GmbHs und A.G.'s aufgenommen werden darf, und dem neuesten Versicherungsgesetz. An der Spitze steht in dieser Gruppe (ähnlich dominierend wie die PZU in bezug auf die Allgemeinheit) die schon erwähnte genossenschaftliche Versicherungsanstalt "Westa" in Lódź, mit ungefähr $2 \%$ der allgemeinen Prämieneinnahmen in Polen.

Vor dem Inkrafttreten des Gesetzes vom 28. 7. 1990, hat das Finanzministerium etwa 40 Genehmigungen zur Betreibung der Versicherungs- und Rückversicherungstätigkeit erteilt. Auf dem Markt ist jedoch die Tätigkeit (genauer gesagt Ansätze dazu) nur von einigen (4-6) Firmen (AG's, genossenschaftlichen Anstalten und GmbHs!) feststellbar. ${ }^{5}$ Die erste Einschätzung der neu entstandenen Firmen weist darauf hin, dass sie :

- mit einem geringen Grundkapital ausgestattet sind (100 Mln, 2-5 Mld Zl);

- wegen der nicht ausreichenden Kapitalausstattung nicht imstande sein werden, selbständig die Versicherung der grösseren wirtschaftlichen Risiken (Industrieversicherungen, technische Versicherungszweige, Haftpflichtversicherungen) ohne Hilfe der Rückversicherer zu übernehmen;

- in den meisten Fällen nicht über das erforderliche know-how und Facherfahrung im Bereich der Versicherung verfügen;

- Kaderprobleme sowohl auf der Manager- als auch auf der Operationsebene zu bewältigen haben;

- über kein entwickeltes Organisationsnetz verfügen, das ihnen den Zutritt zu dem überregionalen Versicherungsmarkt ermöglichen könnte;

- hauptsächlich im Bereich der populären Massenversicherungen (Hausrat-, (Gruppen-)Lebens- und (Gruppen)-Unfallversicherungen) wirken, wobei sie am häufigsten mit kleinen Modifikationen die bisherigen PZU-Versicherungsbedingungen nachbilden. ${ }^{6}$

Im Moment lässt sich kaum über eine Konkurrenz auf dem Versicherungsmarkt sprechen. Überdies ist vorauszusehen, dass ein grosser Teil der angemeldeten Initiativen angesichts der neuen Konzessionierungserfordernisse, die durch das neue Gesetz festgesetzt werden, wieder verschwinden werden, obwohl der Gesetzgeber einen relativ langen, 2jährigen Zeitraum für die Umgestaltung in eine der jetzt erlaubten Rechtsformen (AG, VvaG) erlaubt.

Für die letzte der o.g. Formen, d. h. Versicherungsverein auf Gegenseitigkeit, sind keine besonders gute Entwicklungsperspektiven in Sicht wegen des Mangels an entsprechenden Traditionen (sie wurden vor über 50 Jahren unterbrochen), know-how, Gruppen, die an deren Gründung interessiert wären und nicht zuletzt wegen der fehlenden negativen

\footnotetext{
s Zu den nennenswerten Initiativen auf diesem Gebiet gehören u. a. folgende Neugründungen: Genossenschaftliche Versicherungs- und Rückversicherungsgesellschaft "Polisa" in Warschau, Gescllschaft für Touristische Versicherungen und Rückversicherung "Atu" A.g. in Warschau, Versicherungsanstalt der Finanz- und Wirtschaftskorporation "Pirobu"' in Poznań, Versicherungs- und Rückversicherungsgesellschaft "Varsovia" S.A. in Warschau, Versicherungs- und Rückversicherungsgesellschaft "Polonia" S.A. in Lodz.

${ }^{6}$ Bisherige Versuche, neue Versicherungsprodukte einzuführen, sind fehlgeschlagen oder bleibcn ohne grösscre wirtschaftliche Bedeutung.
} 
Erfahrungen der Abnehmer im Umgang mit anderen Typen der Versicherer. Nur die PZU könnte kraft Gesetzes zu der in der Vorkriegszeit bewährten PZUW-Form (Państwowy Zaklad Ubezpieczeń Wzajemnych) zurückkehren. Solche Entwürfe sind jedoch gegenwärtig nicht mehr Gegenstand ernsthafter Diskussionen.

So kann für die Einschätzung der Angebotsmöglichkeiten im Bereich der einzelnen Versicherungssparten nur eine Analyse des Versicherungsportefeuilles der PZU von Bedeutung sein.

\subsection{Die Struktur des Versicherungsangebotes}

Das aktuelle Angebot der Versicherer auf dem Binnenmarkt umfasst 50-60 Arten und Formen der Versicherungen, wovon nicht alle eine nennenswerte ökonomische Bedeutung haben. ${ }^{7}$

Die überwiegende Mehrheit von ihnen wird - wie schon erwähnt - ausschliesslich von der PZU geführt, ein geringer Teil - gemeinsam mit anderen Anstalten (s. o.), dagegen haben die selbständigen Angebote, die nicht an die PZU-Vorschläge anknüpfen, nur einen inzidentalen Charakter.

Eine Analyse des bisherigen PZU-Portefeuilles von der quantitativen Seite her zeigt ausdrücklich eine wesentliche Rolle von obligatorischen (sog. gesetzlichen) Versicherungen (bisher 9 Arten in der alleinigen Kompetenz der PZU), deren Anteil sich in den letzten Jahren auf einer (unter normalen Marktverhältnissen nicht erreichbaren) Höhe von 50 Prorent $(1988-50,4 \%, 1989-47,1 \%)$ der allgemeinen Sammlung von Versicherungsprämien stabilisiert hat. ${ }^{8}$

In der Struktur der obligatorischen Versicherungen sind Verkehrsversicherungen (Haftpflicht, Unfall, Kasko) vorherrschend : $54 \%$ (und 25\% der allgemeinen Prämiensammlung), ferner landwirtschaftliche Versicherungen : $33,2 \%$ (und $15,7 \%$ der a. P.) und Versicherungen des festen Vermögens der Staatsbetriebe: $12,8 \%$ (und $6.0 \%$ der a.P.); alle Angaben für das Jahr 1989.

In der Gruppe der fakultativen Versicherungen umfassten Vermögensversicherungen $38,1 \%$ (und $20 \%$ der a. P.), Unfallversicherungen $20 \%$ (und $11 \%$ der a. P.), und Personenversicherungen $41,5 \%$ (und $21,8 \%$ der a. P.).

Wenn man eine kurze Einschätzung des Entwicklungsgrades der einzelnen fakultativen Versicherungsarten versuchen möchte, müsste man als gut entwickelt verschiedene Formen der Kollektiv- und Gruppenlebensversicherung (ungefähr 20 Prozent des Anteils an der allgemeinen Prämiensammlung) sowie der Unfallversicherung (ca. $7 \%$ ) bezeichnen, für welche sich der Indikator der Ausnutzung des sog. Versicherungsfeldes auf der Höhe von $85 \%$ (Gruppen-Unfall) bis 99,6\%(!) (Gruppen-Lebensversicherungen) gestaltete. Nur mittelmässig entwickelt waren die Wohnungsversicherungen ( $2 \%$ des Portefeuilles, der Indikator 21,7$)$ und $\mathrm{Kfz}-$ Kasko-Versicherungen $(3,3 \%$ und $50 \%$ ).

Als schwach entwickelt muss man die Haftpflichtversicherungen (aller Art mit Ausnahme der Berufshaftpflicht) $(1,2 \%$ und $5 \%)$ und Individuallebensversicherung $(1,2 \%$ und $9 \%$ ) bewerten. Absolut unentwickelt sind dagegen die Rentenversicherungen $(0,7 \%$ des Portefeuilles, der o.g. Indikator 0,1\%) mit ca. 4.000 Einzelpolicen im Lande.

\footnotetext{
7 Siehe Anhag 1

8 Siehe Anhang 2
} 
Abnchmer der beiden Versicherungssparten (obligatorische, fakultative) aus dem PZU-Angebot waren zu $70 \%$ die sog. Subjekte der nichtvergesellschafteten Wirtschaft (Privatsektor in der Industrie und Landwirtschaft sowie die Bevölkerung) und zu $30 \%$ die vergesellschaftete Wirtschaft.

\subsection{Die möglichen Implikationen der Veränderungen des Gesetzes für das PZU-Versiche- rungsportefeuilles}

Es würde dafür stehen, an dieser Stelle darübcr nachzudenken, welche Konsequenzen die schon früher erwähnte partielle Aufhebung der gesetzlichen Versicherungen (Kürzung von 9 auf 3) und der Entzug des Monopols, dicse Versicherungen auszuüben, für das PZUPortefeuille bringen könuten.

Bestimmte Grössen des Portefeuilles (Stand von 1990) könnten durch dieselbe Anstalt oder Konkurrenz mit fakultativen Versicherungen "bewirtschaftet" werden:

- $100 \%$ der gesetzlichen Versicherungen des festen Vermögens der Staatsbetriebe, das ist $28.949 \mathrm{Mln}$. Zl. (zu ersetzen durch Industrieversicherungen und technische Versicherungszwcige),

- $55 \%$ des Portefeuilles der gesetzlichen landwirtschaftlichen Versicherungen, d. h. 41.176 Mln Zl. der Prämie,

- $50 \%$ des Portefeuilles der gesetzlichen Verkehrsversicherungen, d.h. $60.950 \mathrm{Mln}$. Zl. der Prämie.

Insgesamt macht dies über 1/3 (27\%) des bisherigen Portefeuilles dieser Anstalt aus.

Der Verlust des Monopols im Bereich der übrigen gesetzlichen Versicherungen wird wahrscheinlich nichts an dem aktuellen Zustand ändern und die PZU dürfte für längere Zeit deren einziger Anbieter (wenn sie selbst auf diese Tätigkeit nicht verzichtet) bleiben.

Der Verzicht auf den obligatorischen Charakter eines Teils der Versicherungen kann eine Häufung negativer Erscheinungen zur Folge haben:

- er kann in der ersten Periode (bis 2 Jahre) eine Massenabkehr von denjenigen Versicherungsarten verursachen, die wegen ihrer den Steuern ähnlichen Zwangseinziehung der Versicherungsprämie mit gleichem Widerwillen behandelt wurden (hier vor allem landwirtschaftliche Versicherungen),

- die Verlegung der gegebenen Art des Versicherungsangebotes aus dem Gebiet der obligatorischen Versicherung auf das fakultative verursacht automatisch eine Erhöhung der Prämie infolge einer Steigerung der Bedienungskosten dieser Versicherung.

Es ist also mit einem beträchtlichen Rückgang im Bereich der nicht mehr gesetzlichen Versicherungsarten und dem daraus resultierenden Mangel an Deckung durch den Versicherungsschutz der Risiken, die überwiegend Privatvermögen gefährden, zu rechnen.

Ähnliche Folgen, wenn auch in cincm wahrscheinlich geringeren Massstab, kann das Zurückziehen des Gesetzgebers von der obligatorischen Versicherung des festen Vermögens der Staatsbetriebe hervorrufen.

Dicsc Schwierigkeiten können sich nicht nur aus den Nachfragegründen ergcben. Bei den gegenwärtigen Finanzproblemen der PZU, der Kapitalschwäche anderer Firmen und dem Mangel an inländischer Rückversicherung kann es an der Anstalt fehlen, die sich 
entschliessen würde, diese Art der Versicherung der Industrierisiken zu führen. Dies mag den Prozess der Reprivatisierung des Nationalvermögens (durch eine Aktienemission) verzögern.

\section{Die wirtschaftlich-finanzielle Lage der PZU}

Im Jahre 1989 hat sich die ökonomisch-finanzielle Lage der PZU wesentlich verschlechtert. Man hat zwar eine absolute Zunahme des Volumens der gesammelten Versicherungsprämie im Vergleich zu 1988 um 74\% verzeichnet, aber sie hatte praktisch ausschliesslich inflationären Charakter.

Die (reale) durch die Inflationsrate korrigierte Höhe der gesammelten Versicherungsprämie für die Jahre 1988/89 im Vergleich zu 1985 (100\%) betrug:

$$
\begin{aligned}
& 1985 \text { - 134,1 Mld. Zl. - } 100 \% \\
& 1988 \text { - 116,0 Mld. Zl. - } 86,5 \% \text { - (Nennwert - 275,4 Mld.) } \\
& 1989 \text { - 58,7 Mld. Zl. - } 43,0 \% \text { - (Nennwert - 479,7 Mld.) }
\end{aligned}
$$

Das zeugt von einem beträchtlichen Rückgang der Versicherungen. Er ist umso bedeutender, als man zugleich ein generelles Bilanzdefizit in Höhe von $260 \mathrm{Mld}$. Zl. (das grösste in der Kfz-Versicherung - $220 \mathrm{Mld}$. Zl., ausserdem in den gesetzlichen landwirtschaftlichen Versicherungen, fakultativen Anbau- und Ernteversicherungen und Einbruchdiebstahlversicherungen) verzeichnet hat.

Die PZU hat praktisch ihr Reservekapital verloren ${ }^{9}$ und die versicherungs-technischen Reserven erheblich angerissen. An dieser Stelle ist zu bemerken, dass der Staatshaushalt keine finanzielle Verpflichtungen des staatlichen Versicherers trägt.

Die Gründe dafür - ausser der Inflation, die die ganze Logik des Versicherungsmechanismus bricht ${ }^{10}$ - waren:

- die Notwendigkeit, die Entscheidungen der Finanzorgane des Staates auszuführen, die die bestimmten gesellschaftlichen Zwecke berücksichtigten und nicht immer den ökonomischen Tatsachen Rechnung trugen (Beispiel: die Bestimmung durch das Finanzministerium einer zu niedrig angesetzten Prämienhöhe bei den Verkehrs(Kfz)-Versicherungen, was der PZU Milliardenverluste zugefügt hat),

- es mangelte bisher an der Möglichkeit, zeitweilig freie Finanzmittel effektiv anzulegen (viele Jahre lang existierte die Pflicht, sie im Staatshaushalt als zinsloses oder niedrig verzinstes Depot anzulegen),

- die organisatorischen Unzulänglichkeiten des Versicherers selbst, die zu Missbräuchen seitens der Versicherten führte (z. B. ein ungenügendes System der Überwachung des technischen Zustandes der versicherten Kraftfahrzeuge).

In den letzten Jahren verzeichnete die PZU auch eine ständige Zunahme der Tätigkeitskosten - gerechnet im Verhältnis zu dem gesamten Versicherungsbeitrag $(19807,8 \%$, $198920 \%$ ). Den niedrigsten Indikator haben die gesetzlichen Versicherungen $(9,12 \%$; darunter: Versicherungen des festen Vermögens der Staatsbetriebe 2,3\%!), und den höchsten die Individual-Lebensversicherungen $(39,4 \%)$ sowie die Hausrat- (sog. Wohnungs-)versicherungen $(32,2 \%)$ aufzuweisen.

${ }^{9}$ Die Kapitalbestände der PZU im Jahre 1988 betrugen: 1 . Satzungsfonds - 7,825 Mld. Zl.; Reservefonds 76,8 Mld. Zl.; versicherungstechnische Reserven: 131,6 Mld. Zl.; Entwicklungsfonds: 4, 1 Mld. Zl.

${ }^{10}$ Inflationsindikator für 1989 betrug je nach Informationsquelle $800-1100 \%$ 
Die Beschäftigung hält sich stabil. Der Kaderstand der PZU im Jahre 1989 gestaltete sich wie folgt:

- das direkt mit dem Versicherungsgeschäft verbundene Personal betrug 15.236 Personen (darunter 2124 Personen mit Hochschulausbildung),

- andere Mitarbeiter 1104 Personen.

Die PZU-Zentrale beschäftigte $2,5 \%$ der Belegschaft.

Ausserdem nimmt die PZU die Vermittlungsdienstleistungen von 3972 Vermittlern (Acquisiteuren), die in 4578 Bezirken tätig sind, in Anspruch.

Die Möglichkeiten, das Nachwuchspersonal anzuwerben, sind verhältnismässig beschränkt. Ausbildungskurse werden lediglich an der Technischen Hochschule in Radom, sowie an der Akademie für Wirtschaftswissenschaften in Poznań durchgeführt. Im Zusammenhang mit der geplanten Einschränkung des Umfanges der Versicherungstätigkeit durch den staatlichen Versicherer sind gewisse Kürzungen im Bereich der Beschäftigung absehbar.

Die neue rechtliche und ökonomische Lage der PZU hat seit gewisser Zeit eine breitere Diskussion zum Thema "Neugestaltung" (Neustrukturierung) der Anstalt hervorgerufen. Es wurden zahlreiche Entwürfe und Initiativen angemeldet, wovon die folgenden die grössten Realisierungschancen haben:

- die Umgestaltung der PZU in zwei Aktiengesellschaften (nach dem Spartentrennungsprinzip: Lebensversicherungs-AG, und sonstige Versicherungsarten-AG),

- die Umgestaltung der PZU in einige selbständige Staatsanstalten nach dem Regionalkriterium (5-9).

Trotz der aktuell schwierigen Lage und der geplanten Restrukturierung bleibt die PZU - wie es scheint - der glaubwürdigste Partner für die Zusammenarbeit im Bereich der Versicherung und Rückversicherung auf dem polnischen Markt, vor allem wegen ihrer gut ausgebauten landesweiten Organisationsstruktur sowie der zahlreichen gut ausgebildeten Mitarbeiterkader im operativen Bereich und der erforderlichen Erfahrung auf dem Binnenmarkt.

\section{Die Nachfragesituation im Rahmen der einzelnen Gruppen der Abnehmer von Versicherungsleistungen}

Angesichts der z. Z. eintretenden staatspolitischen Veränderungen in der Volkswirtschaft, die einen Wandel im Bereich der Verhaltensweisen der Allgemeinheit mitbeeinflussen, sowie in Anbetracht dessen, dass gleichzeitig eine grundlcgende Reform des Versicherungssystems, das das Marktmodell an Stelle des zentralisierten staatlichen Monopols einführt, verwirklicht wird, ist cs überaus schwicrig, glaubwürdige quantitative Prognosen im Bereich der Gestaltung der Nachfrage auf dem Versicherungsmarkt Polens in nächster Zukunft vorzustellen. Als Annäherung kann der Vcrsuch eincr quantitativen Analyse des Versichcrungsbewusstscins in den cinzelnen Gruppen der potentiellen Abnehmer von Versicherungsleistungen dienen.

Beginnen wir mit den Staatsbetricben. In der bisherigen Wirtschaftspraxis der sog. sozialistischen Länder wurde der risikointensive Marktumsatz durch cin risikoncutrales Systcm der planmässigen Produktion und Güterverteilung ersetzt.

Der Staat bcstimmte im Zentralplan die Sortimente und Mengen von hergestellten Gütcrn, deren Prcisc sowic die Höhc des Finanzüberschusses ("Gewinn"), der von den einzelnen "Planausführern" ("Bctricbc") crziclt wurdc, und sichcrte den Absatz der 
Produkte. Die wirtschaftliche Existenz der Betriebe war somit nicht von dem "Markterfolg" abhängig. Die Erscheinung des "Wirtschaftsrisikos" existicrte praktisch im Mikromassstab nicht. * Noch mehr, eine niedrige Effektivität der Planwirtschaft verursachte, dass es in fast allen Branchen einen ständigen Überschuss der angemeldeten "Nachfrage" über das bestehende Angebot gab. Dies ermöglichte, jede Menge von den hergestellten Gütern ohne Rücksicht auf ihre Qualität abzusetzen. Die Aufgabe der damals cxistierenden Vcrsichcrungen bestand darin, die Finanzmittcl für die Liquidicrung derjenigen Zufallsschäden zu liefern, die die Ausführung des Plans durch das gegebene Wirtschaftssubjekt verhindern konnten. Bei dem Mangel eines Marktes (der die Zweckmässigkeit der getragenen Kosten vcrifiziert) brauchte die Versicherungsprämie kein wesentliches Element der Produktionskostenkalkulation zu sein. Die für risk management typischen Risikokalkulationen wurden überhaupt nicht aufgenommen.

Der aktuelle "Ökonomisierungsprozess" ("Vermarktung der Wirtschaft") versetztc die Gesamtheit der Wirtschaftssubjekte, darunter auch Staatsbetriebe, in einc völlig neue Lage, u. a. im Bereich des Wirtschaftsrisikos.

Die Erreichung eines Wirtschaftserfolges ist ausschliesslich von der Möglichkeit abhängig, die Produkte auf dem Markt abzusetzen. Eine grundlegende Bedeutung gewinnt die Kostenrechnung (Gewinn- und Verlustrechnung) und das Wirtschaftsrisiko wird zu einem wesentlichen Element diescr Kalkulation (schwächere Effekte können in den Branchen auftreten, wo weiterhin die bisherigen Monopolisten dominieren).

Die Versicherungswirtschaft hat unter diesen Bedingungen zum ersten Mal die Chance, mit dem Versicherungsangebot die wirklichen Bedürfnisse der Wirtschaftssubjekte zu befriedigen.

Die Staatsbetriebe bilden auf Grund ihres grossen Vermögenspotentials (ungefähr $60 \%$ des Wertes des Nationalvermögens) die attraktivste Gruppe der potentiellen Abnehmer von Versicherungsleistungen.

Nach einer Stabilisierung der ökonomischen Lage und einer Übergangszeit, während derer Erfahrungen im Bereich des Wirtschaftsrisikos gesammelt werden können (1-2 Jahre), ${ }^{* *}$ ist eben in dieser Gruppe die grösste Dynamik des Zuwachses des Versicherungsportefeuilles zu erwarten.

Diese Gruppe - insbesondere die kleinen und mittelgrossen Unternehmen - wird in der nächsten Zukunft den Eigentumsumgestaltungen (Reprivatisierung) unterworfen sein. Ihre Risikoempfindlichkeit wird von dem Versicherungsbewusstseins des zukünftigen Besitzers (AG, GmbH, genossenschaftlichc Unternehmen, staatlich-private Unternehmen) abhängen. Die Prozesse sollten generell den Zustand des Versicherungsbewusstseins der Wirtschaftssubjekte positiv beeinflussen.

Mit der "Ökonomisierung" der Verhältnisse zwischen den einzelnen Wirtschaftssubjekten werden sicher die verschiedenen Arten der Haftpflichtversicherung (Allgemeine Betriebshaftpflicht-, Produktehaftpflicht-, Umweltschutz-Haftpflicht-Versicherung) an

\footnotetext{
* Man konnte hier eventuell von einem politischen Risiko der Leitungskader der Betriebe für die Nichterfüllung des Wirtschaftsplanes sprechen. Eine Absicherung dagegen war die Verringerung der angemeldeten Produktionskapazitäten des Betriebes in der Planungsphase.

${ }^{* *}$ u. a. die Mentalitätsveränderungen der Leitungsgremien, eine Stabilisierung des Versicherungsbewusstseins.
} 
Bedeutung gewinnen, angesichts des grossen Schädigungspotential dieser Gruppe. Die bisher nicht ökonomisch selbständigen Unternchmen wurden durch solche Risiken kaum gefährdet. Ähnlich gutc Entwicklungschancen hat dic in Polen noch nicht betriebene Rechtsschutzversicherung.

Die Privatwirtschaft kann in Scgmente untcrteilt werden:

\section{Handwerk, Klein- und Mittelbetriebe}

In der vorhergegangenen Periode (Planwirtschaft) schuf dic ineffiziente Wirtschaftstätigkcit der Staatsbetriebc permanent Angebotslücken auf dem Markt. Diese Situation wurde von den klcinen Privatfirmen ausgenutzt, indem sie - in der Regel bei bescheidener Kapitalausstattung - cine ausserordentlich hohe Rentabilität der Produktion verwirklichten (bis zu einigen hundert Prozent!). Das ökonomische Risiko und das Bedürfnis nach dem Versichcrungsschutz traten demnach nicht so deutlich in Erscheinung.

Gegenwärtig, infolge der Zunahme der Konkurrenz eincrscits (frcie Devisencinfuhr, die Vergrösserung der Zahl der privaten und ausländischen Firmen) und der Abnahme der Kaufkraft der Bevölkerung andererseits, erfolgt eine Stabilisicrung der Rentabilität und Einnahmen dieses Sektors auf einem der Marktverhältnissen entsprechendem Niveau. Das Wirtschaftsrisiko wird zu einem gewichtigen Element der wirtschaftlichen Kalkulation. Es ist somit eine rasche Zunahme des Interesses dieser Gruppe der Subjekte an einem Versicherungsschutz, besonders im Bereich der Vermögens- und Haftpflichtrisiken, vorauszusehen.

\section{Handel und Dienstleistungen}

Der am Anfang d. J. eingeleitete Prozess der sogenannten kleinen Reprivatisierung der Wirtschaft dauert an - auf dem Gebiet des Gross- und Einzelhandels sowie der Dienstleistungen. Ständig und dynamisch wächst die Zahl der privaten Firmen, die im Warenumsatz und in der Vermittlung tätig sind.

Nachdem man auf die obligatorische Versicherungen der Betriebsmittel der Einheiten der vergesellschafteten Wirtschaft verzichtet hat, bestehen gute Bedingungen für die Übernahme der Versicherungen von Bctriebsmittel der Gesamtheit der Wirtschaftssubjekte (der privaten und staatlichen Betriebe). Diese Situation betrifft auch Frachtversicherungen im Transport.

\section{Joint ventures}

Der für die Marktwirtschaft charakteristische Typ des Versicherungsbewusstscins und der Erfahrung im Bcrcich des Risk Managements wird auf polnischem Boden von den ncu cntstchenden Joint venture-Firmen (vor allem mit ausländischer Mehrheitsbeteiligung) vertreten. Der resulticrende Bedarf an Versicherungsschutz wird mit der quantitativen Entwicklung dieser Investitionsform auf dem polnischen Markt und mit der Einbringung eines grösscren Einzelkapitals anwachsen.

Es ist mit eincr erheblichen Zunahme des Bedarfs an professionellem Versicherungsschutz seitens dicscr Gruppe von Wirtschaftssubjekten innerhalb von 2-3 Jahren zu rechnen.

\section{Landwirtschaft}

Nach der im neuen Gesetz vorgesehenen Aufhebung der gesetzlichen landwirtschaftlichen Versichcrungen (gesetzliche Gebäudeversicherung ausgenommen) ab 1. 1. 1991 
kann ein erheblicher Rückschritt in Bezug auf die Zahl der geschlossenen Versicherungsverträge, insbesondere in der Gruppe der Privatbauern, als Reaktion auf eine unpopuläre "finanzielle Belastung" crfolgen.

Diese Situation trifft für die Regionen mit einer hohen landwirtschaftlichen Kultur (Wielkopolska, Kujawy Pomorz), landwirtschaftliche Fachbetriebe und grosse staatliche und genossenschaftliche Produktionsbetriebe nicht $\mathrm{zu}$.

Nach den 2-3 Jahren der "Versicherungsumbildung" ist die Stabilisierung der Versicherungsportefeuilles auf dem bisherigen Niveau möglich. *

\section{Haushalte (Bevölkerung)}

Die Haushalte - im Unterschied zu anderen Wirtschaftssubjekten im System der Planwirtschaft (z. B. Staatsbetriebe) - waren und sind Subjekte, die auf eine natürliche Weise das Risiko und Bedürfnis nach dem Versicherungsschutz empfinden, weil sie über begrenzte Mittel verfügen. Das Niveau des Versicherungsbewusstseins korrespondiert jedoch deutlich mit dem Lebensstandard der Bevölkcrung.

Im Januar 1990 erfolgte in Polen eine drastische Senkung des Niveaus des Individualkonsums (der Ankauf von Waren und Dienstleistungen verringerte sich um 39,3 Prozent) und des Realeinkommens (um 35,6 Prozent im Vergleich zum Januar 1989). Die aktuelle durchschnittliche Struktur der Konsumausgaben beträgt 30-35 Prozent ; ständige Gebühren 5-10 Prozent; andere Ausgaben (Reserven). Trotz den Anzeichen einer Stabilisierung der Finanzlage der Bevölkerung ist vor 2-3 Jahren keine Rückkehr zum Konsumniveau vor dem Januar $1990 \mathrm{zu}$ erwarten.

In dieser Lage muss man mit einem generellen Abflauen des Interesses am Versicherungsschutz in der Präferenzskala der breiten Verbraucherkreise rechnen. Dieses Interesse kann im Bereich der populärsten fakultativen Versicherungen, d. h. der gebundenen Hausratversicherung (bei negativen Verlauf der Schädigungstendenz) sowie der Gruppenlebensversicherungen ${ }^{* *}$ unverändert bleiben.

Es gibt nichtbewirtschaftete Marktnischen im Bereich der Individuallebensversicherungen.

Bisher mangelte es an einer Tradition des langfristigen Sparens ausserhalb der Banken. Zugleich haben wir es mit einem niedrigen Niveau der Individualersparnisse, dem Misstrauen gegenüber der Landeswährung, der Inflation, den unattraktiven Versicherungsbedingungen der PZU usw. zu tun. Im Hinblick auf die angekündigte Reform des Systems der Sozialversicherung (in Richtung der westlichen Länder) ist eine Zunahme der Nachfrage zu erwarten.

Es bestehen potentiell gute Möglichkeiten der Verkaufs von Versicherungsleistungen in Berufsgruppen mit einem hohen Einkommen (immer zahlreichere Kaufleute, Industrielle, Handwerker, Juristen, Manager, Farmer usw.).

\footnotetext{
* Das aktuelle Schädigungsniveau in dieser Versicherungsgruppe bewegt sich zwisehen 41,7115,0 Prozent.

** Eine in den Betrieben populäre, einfache Form der Versicherung für den Todesfall eines Familienmitglieds (17.500 Versicherte). Sie offeriert sehr nicdrige Entschädigungsleistungen (50.0001.000.000 Zl.) auf Grund einer billigen Versicherungsprämie.
} 
Mit einer ähnlichen Situation haben wir es im Bereich der Haftpflichtversicherungen (eine Differenzierung des Angebotes für einzelne Berufs- und Einkommensgruppen vorausgesetzt) sowie der auf unserem Markt nicht auftretenden Rechtsschutzversicherungen zu tun.

\section{Schlussbemerkung und Zusammenfassung}

Im grossen und ganzen ist das Niveau des Versicherungsbewusstseins in Polen als entschieden niedriger im Vergleich zu dem der westlichen Länder und als mittelmässig in der Gruppe der osteuropäischen Länder zu bewerten. Es weist ausserdem ziemlich grosse Schwankungen und territoriale sowie regionale Unterschiede im Verhältnis Stadt - Dorf auf. Über die höchste Versicherungskultur verfügen Wielkopolska, Sląsk, Warszawa, Pomorze, über die niedrigste die Wojewodschaften der Ostzone. durch:

Die Einschätzung des Niveaus des Versicherungsbewusstscins wird deutlich erschwert

- das Fehlen der systematischen Nachfrageforschungen,

- die sich rasch verändernden Bedingungen der Tätigkeit der Wirtschaftssubjekte,

- das Auftauchen neuer Formen von Wirtschaftsobjekten,

- die Eigentumsgestaltungen im Rahmen der bisherigen Staatswirtschaft usw.

Als Zusammenfassung der bisherigen Feststellungen kann gesagt werden, dass der Erfolg der Versicherungsreform in Polen von dem Erfolg und Tempo des Ökonomisierungsprozesses der Wirtschaftsverhältnisse, d.h. der "Vermarktung" und "Privatisierung" der Wirtschaft abhängig ist. Die Schwierigkeit beruht darauf, dass es auf vielen Ebenen vernetzte Prozesse sind.

Es steht dagegen fest, dass die Versicherungen in Polen zum ersten Male seit längerer Zeit zur einer allen Subjekten notwendigen Wirtschaftseinrichtung, zu einem unentbehrlichen Element des wirtschaftlichen Lebens werden.

\section{ANHANG I.}

Liste der Versicherungssparten, die von den polnischen, zur Tätigkeit auf dem Binnenmarkt zugelassenen Versicherern geführt werden

Gesetzliche (obligatorische) Versicherungen, die ausschliesslich von der PZU geführt werden.

Gesetzliche landwirtschaftliche Versicherungen:

1. Gebäude-Feuerversicherung und gegen andere zufällige Ereignisse,

2. Mobilien-Feuerversicherung und gegen andere zufällige Ereignisse,

3. Hagel- und Überschwemmungsversicherung,

4. Landwirthaftpflichtversicherung.

Bis 1. 7. 1989 galten gesetzliche Versicherungen auch für die Tiere. Nach dem Regierungsentwurf des GüPuVv (vom 19. 3. 1990) sollen die in den Punkten 2, 3, 4, enthaltenen Vcrsicherungen aufgehoben werden.

Gesetzliche Verkehrsversicherungen

1. Kfzhaftpflichtversicherung

2. Kfzunfallversicherung

3. Kfzkaskoversicherung 
Nach dem vorher zitierten Entwurf wird dic Aufhebung der in den Punkten 2 und 3 genannten Versicherungen gcplant.

Gesetzliche Versichcrung des festen Vcrmögcns der Staatsbetriebe gegen Feuer und andere zufällige Ereignisse.

Dic Aufhebung der Versicherungspflicht ist vorgesehen.

Obligatorische Atomhaftpflichtversicherung

Obligatorische Haftpflichtversicherung der ausländischen Personen, die in Polen Bau- und Montagearbeiten leisten.

Der vorher genannte Regierungsentwurf sieht keine Möglichkeit vor, die obligatorische Form dieser Versicherung aufrechtzuerhalten.

Es ist zu erwarten, dass dic Aufhebung der oben genannten gesetzlichen Versicherungen ab 1. 7. 1990 erfolgt und ab 1. 1. 1991 in Kraft tritt.

Freiwillige (Fakultative) Versicherungen

Vermögensversicherungen

Landwirtschaftliche Versicherungen: 1. TierV, 2. Anbau- und ErnteV, 3. GeflügelV, 4. Bienenzucht $V, 5$. Teichfischzucht $V, 6$. andere kleine Landwirtschaftliche Versicherungen (z. B. Seidenraupen).

Feuerversicherungen: 1. des Vermögens, 2. der Maschinen gegen Schäden durch Strom, 3. Betriebsunterbrechungsversicherung, 4. gegen Neuerungsrisiken.

Transportversicherungen: 1. Kargo im Binnenverkehr, 2. Kasko in der Binnenschiffahrt, 3. Aerokasko, 4. andere kleine Transportversicherungen (z. B. der Sportboote).

Andere: 1. Diebstahl/Einbruch/Raubversicherung, 2. kombinierte Hausratversicherung, 3. Versicherung der Wochenendhäuser und ihres Vermögens, 4. Glasversicherung, 5. Reisegepäckversicherung.

Unfallversicherung:

Versicherungen gegen Folgen der Unfälle: 1. IndividualV, 2. GruppenV der Belegschaft, 3. GruppenV der Vorschul- und Schuljugend, 4. GruppenV und IndividualV der Sportvereine und Vereinigungen, 5. kurzfristige Unfallversicherung (Ferien, Kuraufenthalte, Reise, Gelegenheitsveranstaltungen).

Haftpflichtversicherungen

1. Allgemeine HaftpflichtV der Betriebe und Behörden, 2. FachV (für verschiedene Berufsgruppen und Tätigkeitsarten), 3. ProdukthaftpflichtV (seit 1. 1. 1989).

Kfzversicherungen:

1. KfzkaskoV, 2. KfzkaskofachV (für Filmteams, rent a car, Rennen usw.), 3. Versicherung gegen zusätzlichc Risiken (besondere Ausrüstung des Kfz usw.).

Kapitalversicherungen

Bankkreditversicherungen

Kapitalversicherung gegen Inflation

(die beiden Arten hat "Westa" seit 1. 1.1989 cingcführt).

Personenversicherungen:

Lebensversicherungen: 1. IndividualtodesfallV, 2. Individual-gcmischte, Todesfall- und ErlebensfallV, 3. KinderversorgungV, 4. Gruppen-FamilienV in Bctrieben, 5. GruppenfortgesetzteV, 6. gegen AIDS (wird von der "Westa" seit 1989 geführt). 
Rentenvcrsicherungen: 1. aufgeschobene Renten (einige Arten), 2. sofort beginnende Renten (einige Arten), 3. GruppenrcntenV in den Betrieben (Allgemeine Versicherungsbedingungen seit 1967 - keine Vcrsicherungsverträgc).

Insgesamt werden in Polcn 50-60 Versicherungsarten geführt, von dencn lediglich cinige von tatsächlicher wirtschaftlicher Bedeutung sind (s. w. die Analyse des PZU-Portefeuilles). Von den oben genannten Versichcrungsarten werden fast alle selbständig von dem staatlichen Versicherer geführt.

Angesichts dieser Tatsache kann eine aktuelle strukturelle und quantitative Analyse ausschliesslich auf Grund der statistischen Matcrialien der PZU vorgenommen werden.

\section{ANHANG II.}

Die Struktur des PZU-Portefeuilles in den Jahren 1988-89 (nach Versicherungsprämien) *

\begin{tabular}{|c|c|c|c|c|c|c|c|c|}
\hline Zahl & Versicherungsart & $\begin{array}{c}\text { Prämie in } \\
\text { Mln. Zl. }\end{array}$ & $\%$ & $\%$ & $\begin{array}{l}\text { Prämie in } \\
\text { Mln. Zl. }\end{array}$ & $\%$ & $\%$ & $\begin{array}{c}\text { Zuwachs } \\
88-89\end{array}$ \\
\hline & Insgesamt & 275,481 & 100 & 100 & 479,725 & 100 & 100 & 174 \\
\hline & 1. Gesetzliche & 138,882 & 50,4 & & 225,768 & 47,1 & & 164,4 \\
\hline & 2. Fakultative & 136,599 & 49,4 & & 253,857 & 52,9 & & 184,7 \\
\hline & 3. Vergesellschaftete W. & 82,386 & 30,0 & & 133,394 & 27,8 & & $161,-$ \\
\hline & 4. Nicht vergesellschaftete $W$. & 193,095 & 70,0 & & 346,231 & 72,2 & & 180,0 \\
\hline & \multicolumn{8}{|l|}{ 5. Gesetzliche: } \\
\hline & VerkehrsV & 66,319 & 24,1 & 47,7 & 121,961 & 25,4 & 54,0 & $18-,-$ \\
\hline & landwirtschaftl.V & 54,935 & 20,5 & 40,8 & 74,866 & 15,7 & 33,2 & $136,-$ \\
\hline & des festen Vermögens & 16,050 & 5,8 & 11,5 & 28,940 & 6,0 & 12,8 & $180,-$ \\
\hline & \multicolumn{8}{|l|}{ 6. Fakultative: } \\
\hline & VermögensV & 46,034 & 16,7 & 33,7 & 96,402 & 20,2 & 38,1 & $200,-$ \\
\hline \multirow[t]{6}{*}{ darunter: } & landwirtschaftl.V & 2,838 & 1,1 & 2,1 & 19,927 & 4,1 & 7,8 & $602, *$ \\
\hline & FeuerV & 16,320 & 5,9 & 11,9 & 27,143 & 5,6 & 10,7 & $163,-$ \\
\hline & HausratV & 9,043 & 3,3 & 6,6 & 22,923 & 4,8 & 9,0 & $25-,-$ \\
\hline & EinbruchdiebstahlV & 5,550 & 2,0 & 4,1 & 9,596 & 2,0 & 3,8 & 17-,- \\
\hline & andere & 12,283 & 4,4 & 9,0 & 16,813 & 3,7 & 6,8 & $136,-$ \\
\hline & UnfallV & 31,920 & 11,7 & 23,4 & 51,678 & 10,9 & 20,3 & $161,-$ \\
\hline \multirow[t]{6}{*}{ darunter: } & GruppenV & 12,480 & 4,5 & 9,1 & 18,284 & 3,8 & 7,2 & $14-,-$ \\
\hline & JugendV & 4,675 & 1,7 & 3,4 & 7,678 & 1,6 & 3,0 & $16-,-$ \\
\hline & KfzkaskoV & 8,877 & 3,2 & 6,5 & 15,184 & 3,2 & 6,0 & $171,-$ \\
\hline & HaftpflichtV & 2,958 & 1,2 & 2,2 & 5,601 & 1,2 & 2,2 & 189,3 \\
\hline & andere & 2,930 & 1,1 & 2,2 & 4,931 & 1,1 & 1,9 & 168,3 \\
\hline & PersonenV & 59,482 & 21,2 & 42,9 & 105,782 & 21,8 & 41,6 & 178,0 \\
\hline \multirow[t]{2}{*}{ darunter: } & Renten & 57,633 & 0,6 & 1,2 & 9,349 & 0,7 & 1,4 & 223,4 \\
\hline & LebensV & 57,849 & 20,6 & 41,7 & 102,133 & 21,1 & 40,4 & 176,5 \\
\hline \multirow[t]{2}{*}{ darunter: } & IndividualV & 3,759 & 1,3 & 2,7 & 6,714 & 1,2 & 2,2 & 152,0 \\
\hline & GruppenV & 54,090 & 19,3 & 39,0 & 96,419 & 19,9 & 38,0 & 182,2 \\
\hline
\end{tabular}

* nach eigener Berechnung 